\title{
Erratum: Helicobacter pylori exploits human CEACAMs via HopQ for adherence and translocation of CagA
}

Verena Königer, Lea Holsten, Ute Harrison, Benjamin Busch, Eva Loell, Qing Zhao, Daniel A. Bonsor, Alexandra Roth, Arnaud Kengmo-Tchoupa, Stella I. Smith, Susanna Mueller, Eric J. Sundberg, Wolfgang Zimmermann, Wolfgang Fischer, Christof R. Hauck and Rainer Haas

Nature Microbiology 2, 16188 (2016); published 17 October 2016; corrected 31 October 2016.

The original version of this Article contained numerous errors in the affiliations, which were introduced during production. These have been corrected in all versions of the Article. We apologise to the authors and our readers for any confusion caused. 